\title{
Visual Experience for Recognising Human Activities
}

\author{
$\mathrm{Na} \mathrm{Li}$, Martin Crane, and Heather Ruskin \\ Centre for Scientific Computing \& Complex Systems Modelling, \\ School of Computing, Dublin City University, Ireland \\ \{na.li\}@dcu.ie \\ \{mcrane, hruskin\}@computing.dcu.ie
}

\begin{abstract}
Technologies for Ambient Assisted Living (AAL) combine new Information and Communication Technologies (ICT) to improve and increase the quality of life of the elderly. The SenseCam visual lifelogging device is now used not only to support memory recall, but also by research groups in other fields in order to investigate human lifestyle. Recent and continuing work in Dublin City University's SCI-SYM centre has been an application and evaluation of a novel approach, namely use of the cross correlation matrix and Maximum Overlap Discrete Wavelet Transform (MODWT) to analyse SenseCam lifelog data streams. By examination of the eigenspectrum, we show that these approaches enable detection of key sources or major events in the time SenseCam recording, with MODWT also providing useful insight on details of major events. In this paper, we analyse the data collected from the EvAAL (Evaluating AAL System Through Competitive Benchmarking) competition. The results confirmed our previous findings $[1,2]$. We believe using this highlight key episodes to identify the boundaries can be used to develop automatic classifiers for visual lifelogs to infer different lifestyle characteristics.
\end{abstract}

Key words: Ambient Assisted Living; SenseCam; time series methods; EvAAL competition

\section{Introduction}

Recently, the numbers of elderly people in developed counties has been increasing dramatically. Ambient Assisted Living (AAL) combines new Information and Communication Technologies (ICT) with the aim of improving the quality of life and health of the older population. EvAAL is an international competition aimed at the evaluation and assessment of Ambient Assisted Living systems, components, services and platforms ${ }^{1}$. In order to evaluate each competing system, the EvAAL competition applies a set of evaluation criteria such as performance, user acceptance, recognition delay, installation complexity and integrability to AAL

\footnotetext{
${ }^{1}$ http://evaal.aaloa.org
} 
systems in order to evaluate and rank AAL applications [3]. In EvAAL 2012, Dublin City University (SCI-SYM) centre used the SenseCam lifelog device to track the activity and recognition in the competition scenario. In this paper, we introduce a time series approach to analyse and evaluate the SenseCam lifelog data, collected for the EvAAL competition.

Developed by Microsoft Research in Cambridge, UK, SenseCam [4] is a small, wearable camera that, along with other sensor data recorded, takes images automatically, in order to document the events of the user's day. It can be periodically reviewed by the user to refresh and strengthen memory of an event. Besides a camera, the SenseCam also contains several electronic sensors. including those which record light-intensity and light-colour, a passive infrared (body heat) detector, a temperature sensor, and a multiple-axis accelerometer for monitoring changes in movement in the $X, Y, Z$ directions of the user's environment. The device takes pictures at VGA resolution, (480x640 pixels), and stores these as compressed JPEG files on internal flash memory $(1 \mathrm{~Gb})$. It can collect a large amount of data, even over a short period of time. Since SenseCam typically takes a picture every 30 seconds, thousands of images are captured per day. Experience shows that the SenseCam can be an effective memory-aid device $[5,6]$, as it helps users to improve retention of an experience. SenseCam is now used to not only support memory recall, but also by research groups [7] in other fields to investigate human lifestyle. The challenge is to manage, organize and analyse these large image collections in order to automatically highlight key episodes in the wearer's life.

In terms of this analysis, we note that in recent years, the role of the largest Eigenvalue of a cross-correlation matrix over small windows of time, has been studied extensively, e.g. for financial series [8-11], electroencephalographic (EEG) recordings $[12,13]$, magnetoencephalographic (MEG) recordings [14] and a variety of other multivariate data. In this paper, we apply the same approach to analyse SenseCam lifelog data streams. We aim to apply the multiscaled crosscorrelation matrix technique to study the dynamics of the SenseCam images, where this time series should exhibit atypical or non-stationary characteristics, symptomatic of "Distinct Significant Events" in the data. We can use such highlighted key episodes to identify the boundaries between different daily events. These might include the wearer working in the office, walking outside, shopping etc. We found that different distinct events or activities can be detected at different scales $[1,2]$. In this context, we expected analysis of data collected from EvAAL to confirm previous findings.

This paper is organized as follow: in Section 2, we review the methods to be used in the paper, in Section 3 we describe the data used, while Section 4 details the results obtained. Finally the conclusion is followed by details of future work to be preformed. 


\section{Methods}

Our previous research [1] has shown that SenseCam image time series reflect strong long-range correlation, indicating that the time series is not a random walk $^{2}$, but is cyclical, with continuous low levels of background information picked up constantly by the device. In this section, we first use equal-time crossCorrelation Matrices to characterise dynamical changes in non-stationary multivariate SenseCam time-series. The Maximum Overlap Discrete Wavelet Transform (MODWT) is then used to calculate equal-time Correlation Matrices over different time scales. This enables exploration of details of the Eigenvalue spectrum and in particular, examination of whether specific events show evidence of distinct signatures at different time scales.

\subsection{Correlation Dynamics}

The equal-time cross-correlation matrix can be used to characterise dynamical changes in non-stationary multivariate time series.

Given pixels $G_{i}(t)$, of a collection of images, we normalize $G_{i}$ within each window in order to standardize the different pixels for the images as follows:

$$
g_{i}(t)=\frac{G_{i}(t)-\overline{G_{i}(t)}}{\sigma_{(i)}}
$$

where $\sigma_{(i)}$ is the standard deviation of $G_{i}$ for image numbers $i=1, \ldots, N$, and $\overline{G_{i}}$ is the time average of $G_{i}$ over a time window of size $T$. Then the equal-time cross-correlation matrix may be expressed in terms of $g_{i}(t)$

$$
C_{i j} \equiv\left\langle g_{i}(t) g_{j}(t)\right\rangle
$$

The elements of $C_{i j}$ are limited to the domain $-1 \leq C_{i j} \leq 1$, where $C_{i j}=$ 1 defines perfect positive correlation, $C_{i j}=-1$ corresponds to perfect negative correlation and $C_{i j}=0$ corresponds to no correlation. In matrix notation, the correlation matrix is expressed as $C=\frac{1}{T} G G^{t}$, where $G$ is an $N X T$ matrix with elements $g_{i t}$.

The Eigenvalues $\lambda_{i}$ and eigenvectors $\bar{v}_{i}$ of the correlation matrix $C$ are found from the Eigenvalue equation $C \overline{v_{i}}=\lambda_{i} \overline{v_{i}}$ and then ordered according to size, such that $\lambda_{1} \leq \lambda_{2} \leq \ldots \leq \lambda_{N}$. Given that the sum of the diagonal elements of a matrix (the Trace) remains constant under linear transformation, $\sum_{i} \lambda_{i}$ must always equal the trace of the original correlation matrix. Hence, if some eigenvalues increase then others must decrease, to compensate, and vice versa (Eigenvalue Repulsion).

\footnotetext{
${ }^{2}$ A random walk is a mathematical formalization of a path that consists of a succession of random steps.
} 


\subsection{Wavelet Multiscale analysis}

The Maximum Overlap Discrete Wavelet Transform (MODWT) [15-18], is a linear filter that transforms a series into coefficients related to variations over a set of scales. It produces a set of time-dependent wavelet and scaling coefficients with basis vectors associated with a location $t$ and a unitless scale $\tau_{j}=2^{j-1}$ for each decomposition level $j=1, \ldots, J_{0}$. Unlike the DWT, the MODWT, has a high level of redundancy. However, it is non-orthogonal and can handle any sample size $N$, whereas the DWT restricts the sample size to a multiple of $2^{j}$. MODWT retains downsampled ${ }^{3}$ values at each level of the decomposition that would be discarded by the DWT. This reduces the tendency for larger errors at lower frequencies when calculating frequency dependent variance and correlations, as more data are available.

Decomposing a signal, using the MODWT to $J$ levels, theoretically involves the application of $J$ pairs of filters. The filtering operation at the $j^{t h}$ level consists of applying a rescaled father wavelet to yield a set of detail coefficients

$$
\tilde{D}_{j, t}=\sum_{l=0}^{L_{j}-1} \tilde{\varphi}_{j, l} f_{t-l}
$$

and a rescaled mother wavelet to yield a set of scaling coefficients

$$
\tilde{S}_{j, t}=\sum_{l=0}^{L_{j}-1} \tilde{\phi}_{j, l} f_{t-l}
$$

for all times $t=\ldots,-1,0,1, \ldots$, where $f$ is the function to be decomposed [19]. The rescaled mother, $\tilde{\varphi}_{j, t}=\frac{\varphi_{j, t}}{2^{j}}$, and father, $\tilde{\phi}_{j, t}=\frac{\varphi_{j, t}}{2^{j}}$, wavelets for the $j^{t h}$ level are a set of scale-dependent localized differencing and averaging operators and can be regarded as rescaled versions of the originals.

The wavelet variance $\nu_{f}^{2}\left(\tau_{j}\right)$ is defined as the expected value of $\tilde{D}_{j, t}^{2}$ if we consider only the non-boundary coefficients. An unbiased estimator of the wavelet variance is formed by removing all coefficients that are affected by boundary conditions and is given by

$$
\nu_{f}^{2}\left(\tau_{j}\right)=\frac{1}{M_{j}} \sum_{t=L_{j}-1}^{N-1} \tilde{D}_{j, l}^{2}
$$

where $\tilde{D}_{j, l}$ is a rescaled father wavelet, which yields a set of scaling coefficients, $M_{j}=N-L_{j}+1$ is the number of non-boundary coefficients at the $j^{t h}$ level.

The wavelet covariance between functions $f(t)$ and $g(t)$ is similarly defined to be the covariance of the wavelet coefficients at a given scale. The unbiased

\footnotetext{
${ }^{3}$ Downsampling or decimation of the wavelet coefficients retains half of the number of coefficients that were retained at the previous scale. Downsampling is applied in the Discrete Wavelet Transform
} 
estimator of the wavelet covariance at the $j^{\text {th }}$ scale is given by

$$
\nu_{f g}\left(\tau_{j}\right)=\frac{1}{M_{j}} \sum_{t=L_{j}-1}^{N-1} \tilde{D}_{j, l}^{f(t)} \tilde{D}_{j, l}^{g(t)}
$$

The MODWT estimate of the wavelet cross-correlation between functions $f(t)$ and $g(t)$ may be calculated using the wavelet covariance and the square root of the wavelet variance of the functions at each scale $j$. The MODWT estimator, of the wavelet correlation is thus given by

$$
\rho_{f g}\left(\tau_{j}\right)=\frac{\nu_{f g}\left(\tau_{j}\right)}{\nu_{f}\left(\tau_{j}\right) \nu_{g}\left(\tau_{j}\right)}
$$

where $\nu_{f g}\left(\tau_{j}\right)$ is the covariance between $f(t)$ and $g(t)$ at scale $j, \nu_{f}\left(\tau_{j}\right)$ is the variance of $f(t)$ at scale $j$ and $\nu_{g}\left(\tau_{j}\right)$ is the variance of $g(t)$ at scale $j$.

The multiscaled cross-correlation matrix technique is adopted in order to help highlight non-stationary events at various different granularities (in SenseCam lifelog data streams), which could be of importance.

\section{Data}

In this study, the data were generated from the EvAAL competition. In 2012 EvAAL, the activity recognition track took place in CIAmI Living Lab located on the industrial park, Valencia, Spain. The CIAmI Living Lab is an approximately $90 \mathrm{~m}^{2}$ infrastructure that simulates the real environment of a citizen's home combined with provision of Information and Communication Technologies (ICT) massively distributed across the physical space, but as invisible as possible to the people occupying it as shown (Figure 1). In this work, the data are collected by one user (the actor), wearing the SenseCam over two experiments. Total duration of collection was approximately 13 minutes, forming a total lifelog collection of 66 images, with average capture time of 5 images per minute. As the data capture time was limited, the SenseCam camera capture rate was changed in order to generate as many images as possible during the competition. Figure 2 shows some examples of SenseCam images.

\section{Results}

\subsection{Eigenvalue Dynamics}

From our previous studies [1,2], we selected the least asymmetric (LA) wavelet, (known as the Symmlet, [19]), which exhibits near symmetry about the filter midpoint. LA filters are defined in even widths and the optimal filter width is dependent on the characteristics of the signal and the length of the data series. The $j^{\text {th }}$ level equivalent filter coefficients have a width $L_{j}=\left(2^{j}-1\right)(L-1)+$ 1 , where $L$ is the width of the $j=1$ base filter. In practice the filters for 


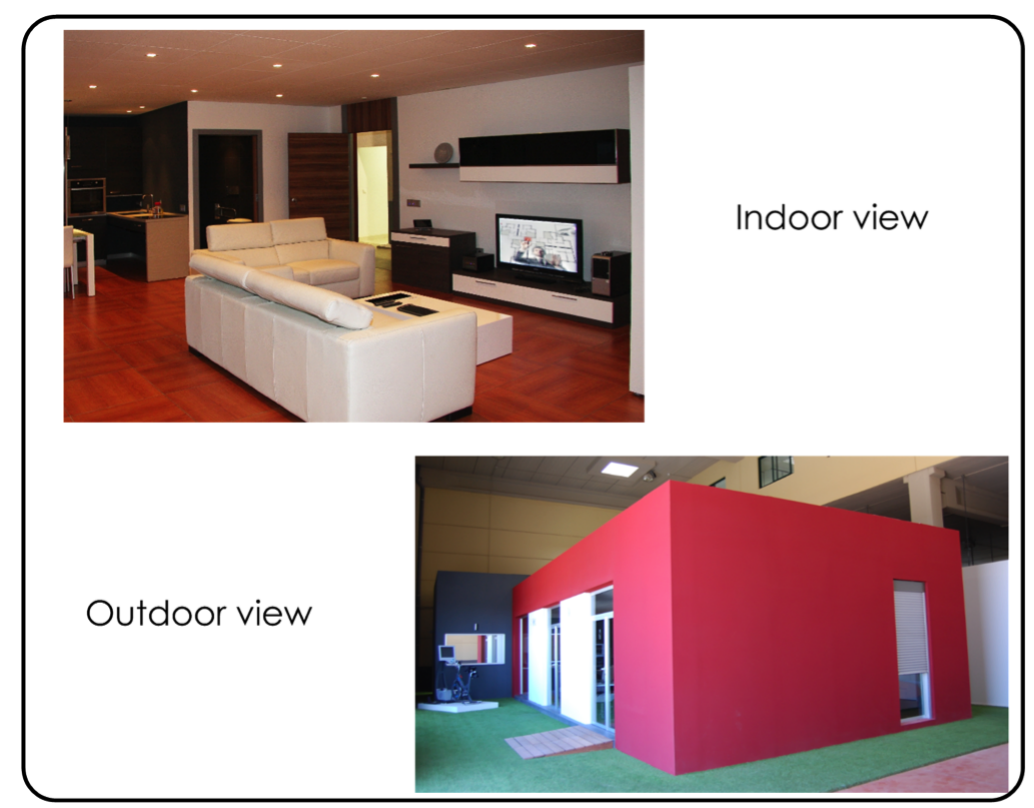

Fig. 1. Indoor and outdoor views of the CIAmI Living Lab

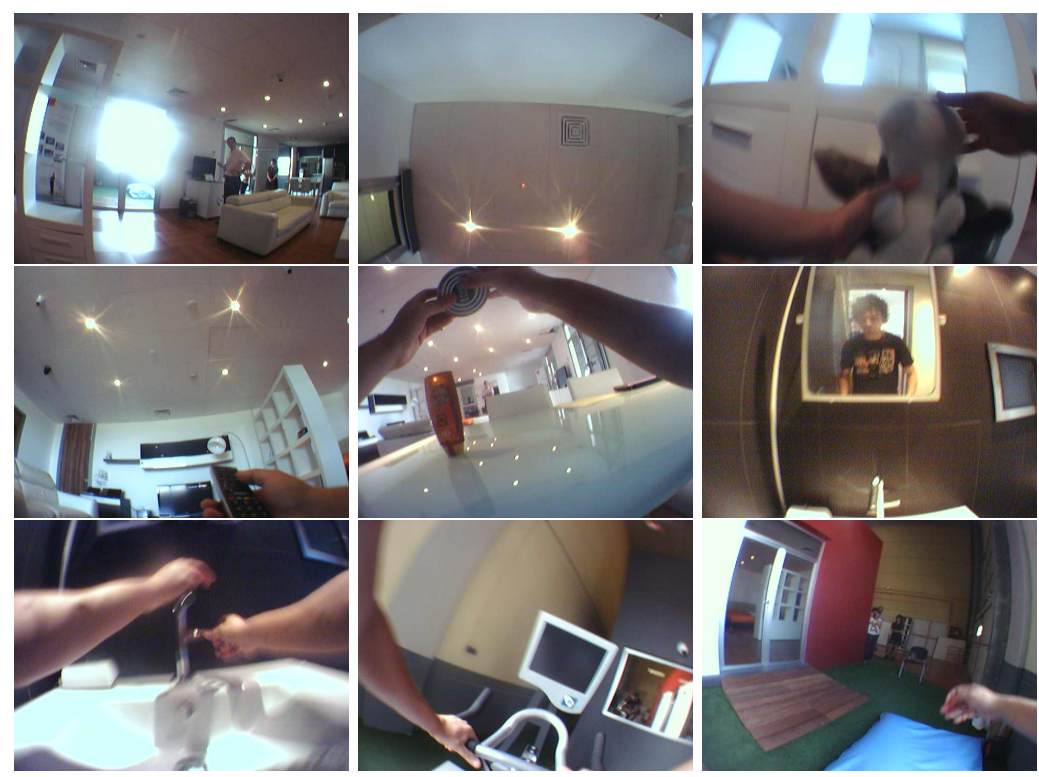

Fig. 2. Example of SenseCam Images 
$j>1$ are not explicitly constructed because the detail and scaling coefficients can be calculated, using an algorithm that involves the $j=1$ filters operating recurrently on the $j^{\text {th }}$ level scaling coefficients, to generate the $j+1$ level scaling and detail coefficients [20]. The filter width chosen for this study was the Haar ${ }^{4}$, since this enables accurate calculation of wavelet correlations to the $5^{\text {th }}$ scale, which is appropriate given the length of data series available. Although the MODWT can accommodate any level, $J_{0}$, the largest level, is chosen in practice, so as to prevent decomposition at scales longer than the total length of the data series, hence the choice of the $5^{t h}[20]$, while still containing enough detail to capture subtle changes in the signal.

Before examining the image time series in detail, it is important to introduce the gray scale pixel values concept. In a gray scale image, a pixel with a value of 0 is completely black and a pixel with a value of 255 is completely white. The images captured from SenseCam are coloured and in order to simplify the calculation, the images are converted to gray-scale images.

To reduce the size of the calculation, we first adopted an averaging method to decrease image size from $480 \times 640$ pixels to $3 \times 4$ pixels. We analysed the equaltime cross-correlation dynamics between each image pixels using a sliding window 12 images. This window was chosen such that $Q=\frac{T}{N}=1.25$, thus ensuring that the data would be close to non-stationary in each sliding window (Different values of $Q$ were examined, with little variation in the results). First, the MODWT of each image pixels was calculated within each window and the correlation matrix between pixels at each scale found. The eigenvalues of the correlation matrix in each window were determined, and eigenvalue time series were normalised in time. During the experiment, SenseCam captured five images every minute, so we can measure wavelet eigenvalue dynamics in time (minutes). These results are shown in Figure 3 and discussed below.

Eigenvalue Dynamics at various wavelet scales In financial data, it has been known for some time that the largest eigenvalue $\left(\lambda_{1}\right)$ contains information on risk associated with the particular assets of which the covariance matrix is comprised, (i.e. the 'market' factor) [20,21]. Similarly we would expect the largest eigenvalue here to present information from the image that reflects the largest change in the SenseCam recording. We also wish to ascertain whether the sub-dominant eigenvalues $\lambda_{2}, \lambda_{3}$, etc hold further information on the key sources or major events and what information these contribute in addition $\lambda_{1}$ to the images. The dynamics of the largest eigenvalue and changes of ratio eigenvalues were examined by the MODWT analysis.

We have studied the largest eigenvalue $\lambda_{1}$ time series for a window size of 12 images to try identify the position and nature of peak sources or major events from the real images generated from SenseCam.

In Figure 3, the dynamics of the series for the largest eigenvalue were examined from the MODWT analysis. The technique gives a clear picture of the movements in the image time series by reconstructing them using each wavelet

\footnotetext{
${ }^{4}$ The $j^{\text {th }}$ level equivalent filter coefficients have a width $2^{j}$
} 

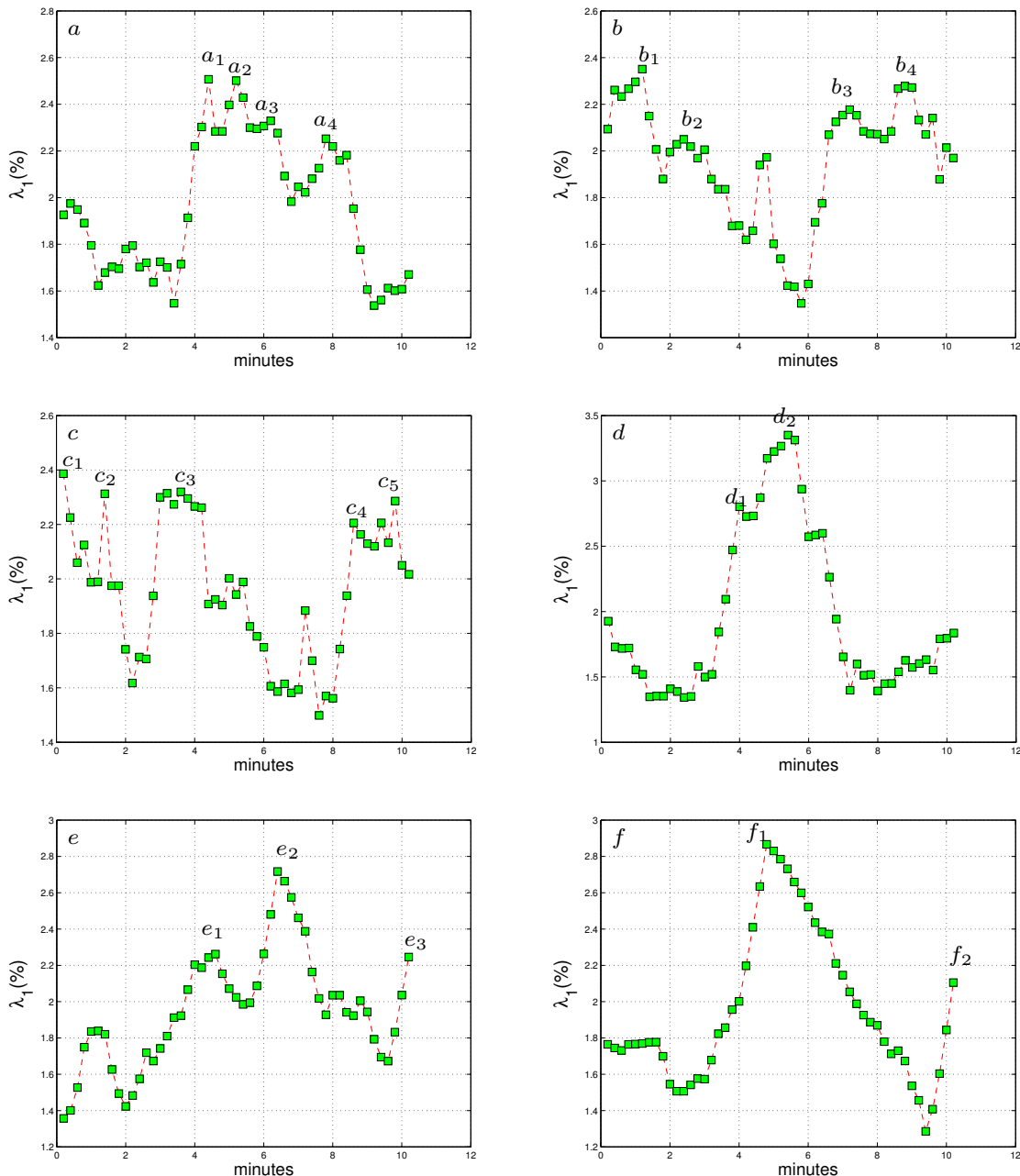

Fig. 3. (a) The Largest Eigenvalue $\lambda_{1}$ dynamics original data, (b) 30 seconds scale, (c) 1 minute scale, (d) 1.5 minutes, (e) 3 minutes and (f) 6.5 minutes.

Table 1. Activity Trait Analysis

\begin{tabular}{lc}
\hline Activities & Wavelet Scales \\
\hline From Outdoor to Indoor & $a_{1} \& e_{1} \& f_{1}$ \\
From Standing to Lying & $a_{2} \& d_{2}$ \\
From Bending to Walking & $a_{3} \& e_{2}$ \\
From Walking to Sitting & $b_{1} \& c_{2}$ \\
From Indoor to Outdoor & $b_{4} \& c_{4}$ \\
From Falling to Standing up & $c_{3} \& d_{1}$ \\
\hline
\end{tabular}



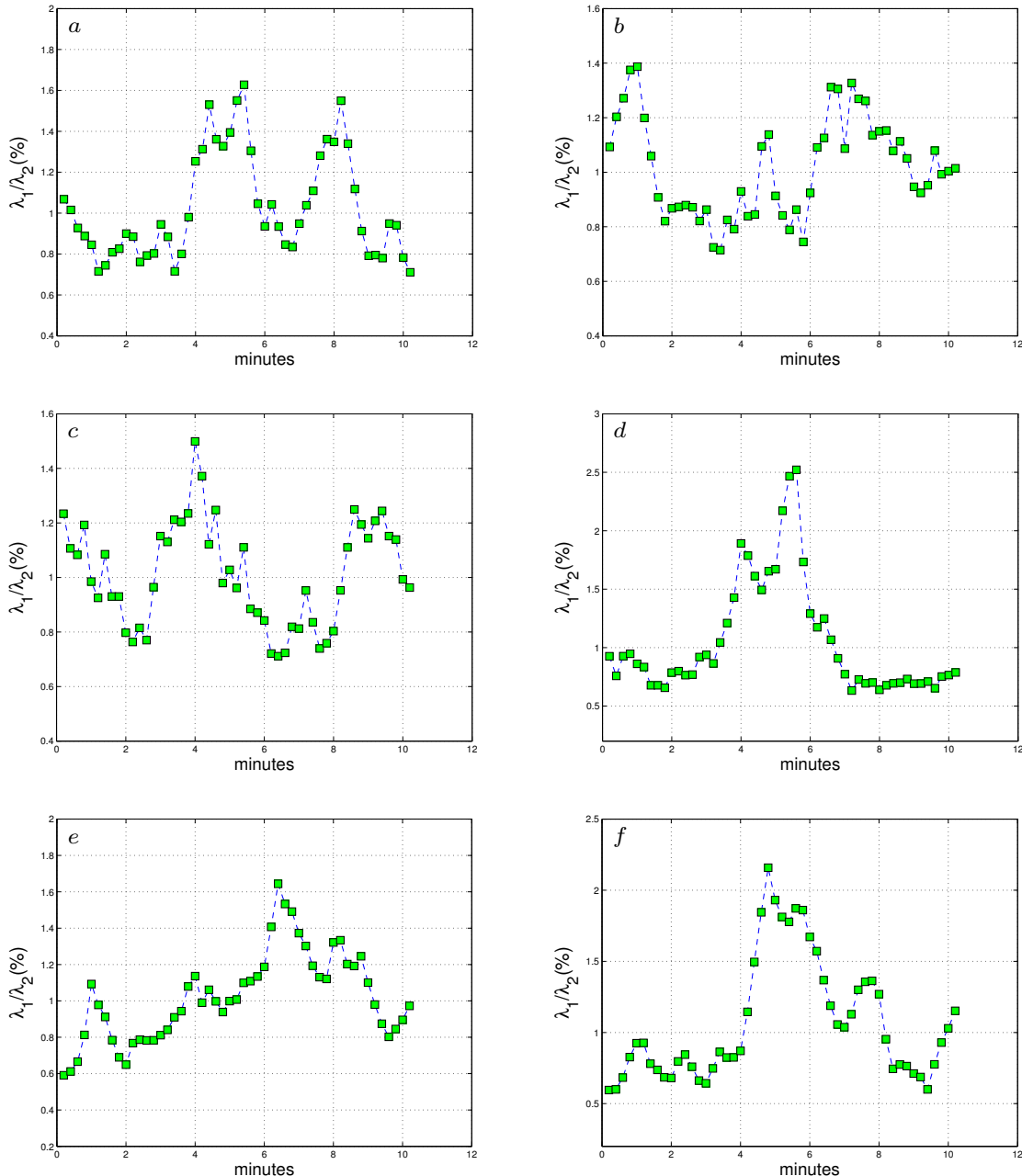

Fig. 4. (a) The ratio of $\lambda_{1} / \lambda_{2}$ dynamics original data, (b) 30 seconds scale, (c) 1 minute scale, (d) 1.5 minutes, (e) 3 minutes and (f) 6.5 minutes. 

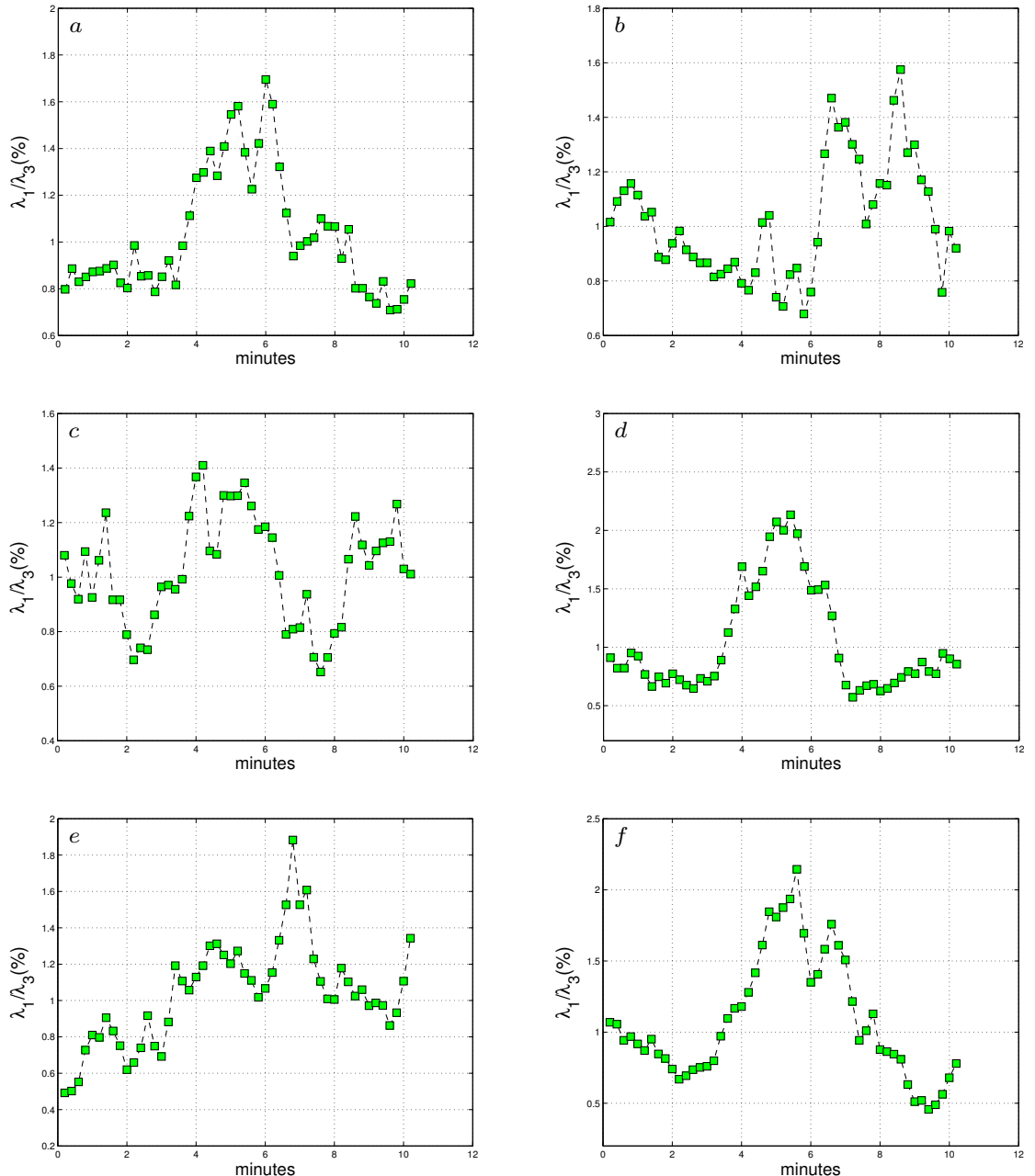

Fig. 5. (a) The ratio of $\lambda_{1} / \lambda_{3}$ dynamics original data, (b) 30 seconds scale, (c) 1 minute scale, (d) 1.5 minutes, (e) 3 minutes and (f) 6.5 minutes. 
component. The MODWT captured the particular features markedly apparent at specified scales. A number of features from the image are reproduced and can be examined by studying these eigenvalue series. Table 1 shows the different features found at various scales, suggesting that the correlation matrix captured different major events with different time horizons. For example, the user moving from walking to sitting in the living room. When the user is sitting at the table, the camera captures the light on the ceiling. Thus, the lights and unchanged 'seated position' contribute higher pixel values in these sequences of images. These changes are captured by wavelet scale 1,2 (peaks $b_{1} \& c_{2}$ ) and correspond to a 30 seconds and 1 minute period. These peaks at different (representative) timescale(s) should help us to identify major events or activities in the data. By examining the dynamics of the largest eigenvalue, we can see that changes in lights, strong sunshine and the subject seating position unchanged over an extended period, contribute to high pixel values in a sequence of images. This confirmed our previous findings. This typical case was always marked by a peak in the SenseCam signal. Similarly it can be seen that the other major events in Table 1 correspond to eigenvalue signal fluctuations visible elsewhere in Figure 3, for example the subject moving from indoors to outdoors, changing light levels, the subject changing position from sitting to moving, movement increase etc.

Above, we have shown that the largest eigenvalue $\lambda_{1}$ contains information on major events captured by the SenseCam. Here, we wish to ascertain whether the subdominant eigenvalues $\left(\lambda_{2}, \lambda_{3}\right)$ hold further information on the key sources or major events and these information contribute additionally to the images. For Figure 4 and 5 , we see the $\lambda_{2}$ and $\lambda_{3}$ have some variations compared with Figure 3. This would seem to imply that the second and third largest eigenvalue carries additional information to describe the images. But both eigenvalues don't contribute in large part to the major events for SenseCam, but do appear to carry information for events surrounding the major ones, e.g. possible lead-in, lead-out $[2]$.

\section{Conclusions and Future Work}

In this paper, we employed initially a time series method in order to highlight key episodes in identification of the boundaries on classification of event type in SenseCam data streams. The Maximum Overlap Discrete Wavelet Transform (MODWT), involving calculation of equal-time correlation matrices over different time scales, was used to investigate the largest eigenvalue and the changes in the sub-dominant eigenvalue ratio spectrums, (Figure 3, with the different features, found at various scales, shown in Table 1). This suggests that the correlation matrix for different information captured by the SenseCam can be filtered by different time horizons. These consistently occurring peaks should help us to identify major events captured by the SenseCam. By examining the behaviour of the largest eigenvalue and the change in the eigenvalue ratios over time, the eigenvalue ratio analysis confirmed that the largest eigenvalue carries most of the major event information, whereas subsequent eigenvalues could carry informa- 
tion on supporting or lead in/ lead out events. On analysing major events, (with a sliding window set to 12 images), we identified light level as an important event delineator during static periods of image sequence. It is possible to identify the time series fluctuation caused by single type change, e.g. with the environment mostly constant, but with the subject movement increased. It is also possible to confirm that the largest time series effects are due to 'grouped' or combined changes, e.g. when the subject's environment is totally changed including light level, introduction of other people and so on. The change in eigenvalue ratios obtained using MODWT provides results in good agreement with those found for the largest eigenvalues. This indicates that the MODWT method may prove a powerful tool for examination of the layered nature of the captured SenseCam data at different time scales. Although the data sets, generated from EvAAL competition were quite limited, some confirmation of some confirmation of our initial findings was obtained, only with a far more challenging dataset; after all the length of the datastream collected during the EvAAL competition didn't permit the extent of multiresolution analysis possible for our earlier work $[1,2]$ was obtained.

In future, investigation of the value of other data sources towards activity recognition would be indicated. The accelerometer could be as an important option in this respect, as has been shown in $[22,23]$. Motion of the SenseCam is calculated using the 3 -axis accelerometer data captured by the device. By analysing acceleration data, we can easily combine contributes from the three different axes by using standard algebra $\left(\sqrt{X^{2}+Y^{2}+Z^{2}}\right)$. Inference of contextual information about common daily activities such as sitting, walking, driving and lying should then be possible from these data also. The combination of the accelerometer and image sensor datastreams together may enable more accurate event boundary identification for life logging data. Larger values for movement indicate that the associated image is quite likely to represent a boundary between different events or activities such as walking from home to work, walking from the office to lunch, walking from home to the shops, etc. So that combining sensor information may help to classify SenseCam images into more meaningful activities. Possible methods include employing different such algorithms as SVM (Support Vector Machines) for example. Activity recognition is a core requirement for Ambient Assisted Living systems.

\section{Acknowledgements}

NL would like to acknowledge generous support from the Sci-Sym Centre Small Scale Research Fund.

\section{References}

1. N. Li, M. Crane and H. J. Ruskin: Automatically detecting "significant events" on sensecam. ERCIM News (87) (2011) 
2. N. Li, M. Crane, H. J. Ruskin and C. Gurrin: Multiscaled Cross-Correlation Dynamics on SenseCam Lifelogged Images. MMM 2013. Part I, LNCS, vol. 7732, pp. 490-501. Springer, Heidelberg (2013)

3. S. Chessa, F. Furfari, F. Potorti, P. Barsocchi, M-R. Tazari and R. Wichert: Evaluating AAL System Through competitive Benchmarking (EvAAL) - Technical Aspects of The First Competition Partnerships for Social Innovation in Europe, Proceedings of the AAL Forum (2011) 617-623

4. S. Hodges, L. Williams, E. Berry, S. Izadi, J. Srinivasan, A. Butler, G. Smyth, N. Kapur and K. Wood: SenseCam: A Retrospective Memory Aid, In UbiComp: 8th International Conference on Ubiquitous Computing, volume 4602 of LNCS, California, USA, (2006) 177-193.

5. R. Harper, D. Randall, N. Smyth, C. Evans, L. Heledd and R. Moore.: Thanks for the Memory, In HCI 2007-Proceedings of the 21st BCS HCI Group Conference, Lancaster, U.K., (2007) 39-42

6. R. Harper, D. Randall, N. Smyth, C. Evans, L. Heledd and R. Moore.: The Past is a Different Place: They Do Things Differently There, In Designing Interactive Systems, Cape Town, South Africa, (2008) 271-280

7. A. R. Doherty and A. F. Smeaton: Automatically segmenting lifelog data into events, in WIAMIS: 9th International Workshop on Images Analysis for Multimedia Interactive Services, IEEE Computer Society, Washington, DC, USA, (2008) 20-23

8. V. Plerou, P. Gopikrishnan, B. Rosenow, L.A. Nunes Amaral and H.E. Stanley.: Universal and non-uiversal properties of cross-correlations in financial time series, Phys. Rev. Lett. 83(7) (1999) 1471-1474

9. S. Sharifi, M. Crane, A. Shamie and H.J. Ruskin: Random matrix theory for portfolio optimization: A stability approach, Physica A 335 (3-4) (2004) 629-643

10. T. Conlon, H.J. Ruskin and M. Crane.: Random matrix theory and fund of funds portfolio optimisation, Physica A 382 (2) (2007) 565-576

11. T. Conlon, H.J. Ruskin and M. Crane: Wavelet multiscale analysis for Hedge Funds: Scaling and Strategies, Physica A (2008) 5197-5204

12. K. Schindler, H. Leung, C. E. Elger and K. Lehnertz.: Assessing seizure dynamics by analysing the correlation structure of multichannel intracranial EEG, Brain 130 (2007) 65-77

13. K. Schindler, C.E. Elger and K. Lehnertz: Increasing synchronization may promote seizure termination: Evidence from status epilepticus, Clin, Neurophysiol. 118 (9) (2007) 1955-1968

14. J. Kwapien, S. Drozda and A.A. Ionannides: Temporal correlations versus noise in the correlation matrix formalism: An example of the brain auditory response, Phys. Rev. E 62 (2000) 5557-5564

15. C.-K. Peng, S.V. Buldyrev, S. Havlin, M. Simons, H.E. Stanley and A.L. Golderberger.: On the mosaic organization of DNA sequences, Phys. Rev. E 49 (1994) $1685-1689$

16. J.A.O. Matos, S.M.A. Gama, H.J. Ruskin, A.A. Sharkasi and M. Crane: An econophysics approach to the Portuguese Stock Index-PSI-20, Physica A 342 (2004) 665-676

17. S.V. Buldyrev, A.L. Goldberger, S. Havlin, R.N. Mantegna, M.E. Matsa, C.-K. Peng, M. Simons and H.E. Stanley: Long-range correlation properties of coding and noncoding DNA sequences: GenBank analysis, Phys. Rev. E 51 (1995) 50845091 
18. C. Heneghan and G. McDarby: Establishing the relation between detrended fluctuation analysis and power spectral density analysis for stochastic processes, Phys. Rev. E 62 (2000) 6103-6110

19. C.S. Burrus, R.A. Gopinath and H. Gao: Introduction to wavelets and wavelets transforms, Prentice Hall, (1997)

20. D.B. Percival and A.T. Walden: Wavelet methods for time series analysis, Cambridge University press, (2000)

21. A. Sharkasi, M. Crane, H.J. Ruskin and J.A. Matos: The reaction of stock markets to crashes and events: A comparison study between emerging and mature markets using wavelet transforms, Physica A 368, (2006) 511-521

22. C. O Conaire, N. O'Connor, A. F. Smeaton and G.J.F. Jones: Organising a Daily Visual Diary Using Multi-Feature Clustering, SPIE Electronic Imaging- Multimedia Content Access: Algorithms and Systems. San Jose, California, USA, (2007)

23. A. R. Doherty, A. F. Smeaton, L. Keansub and E. Daniel: Multimodal segmentation of lifelog data. In: RIAO 2007 - Large-Scale Semantic Access to Content (Text, Image, Video and Sound), 30 May - 1 June 2007, Pittsburgh, PA, USA. (2007) 21-38 